\title{
COVID-19 vaccine trial ethics once we have efficacious vaccines
}

\author{
By David Wendler, ${ }^{1}$ Jorge Ochoa, ${ }^{1}$ Joseph Millum, ${ }^{1,2}$ Christine Grady, ${ }^{1}$ Holly A. Taylor ${ }^{1}$ \\ ${ }^{1}$ Department of Bioethics, National Institutes of Health (NIH) Clinical Center, Bethesda, MD, USA. ${ }^{2}$ Fogarty International Center, NIH, Bethesda, MD, USA. \\ Email: dwendler@nih.gov
}

\section{Some placebo-controlled trials can continue ethically after a candidate vaccine is found to be safe and efficacious}

The unprecedented effort to identify one or more safe and effective vaccines for COVID-19 includes more than 180 candidates in development (1), with at least 12 in phase 3 trials (2). The testing of so many vaccine candidates, in a pandemic of a disease for which there are to date limited treatment options, raises a critical challenge: What should researchers do if a vaccine candidate is judged to be safe and efficacious? Guidance from the U.S. Food and Drug Administration (FDA) states that in the event that a COVID-19 vaccine candidate is judged to be "safe and effective," discussion may be necessary "to address ethical arguments to break the blind and offer vaccine to placebo recipients" (3). We consider here two questions raised by this guidance: First, if a vaccine candidate is found to be safe and efficacious in a placebo-controlled trial, should the researchers continue that trial as designed? Second, should researchers continue to test other vaccine candidates using placebo-controlled trials? These two questions are especially timely given recent announcements by Pfizer and Moderna that their vaccine candidates have been found to be efficacious in preventing symptomatic COVID-19 $(4,5)$.

\section{VACCINE SAFETY AND EFFICACY}

A finding of efficacy occurs when a vaccine candidate being tested in a phase 3 trial meets its efficacy end point, either during an interim analysis or after study completion. The primary efficacy end point of current phase 3 trials is whether the vaccine candidate prevents symptomatic COVID-19 in individuals newly infected with severe acute respiratory syndrome coronavirus 2 (SARS-CoV-2) (6). A finding of efficacy in these trials thus implies that the vaccine candidate protects infected individuals from experiencing symptoms. A finding of safety occurs when there is sufficient data on the impact of the vaccine candidate to conclude that its potential benefits justify its risks in the target population.

A single finding of safety and efficacy may not be sufficient for a vaccine candidate to receive FDA approval. Depending on the strength of the data, it may be important to conduct additional research to learn more about the vaccine candidate and gain greater confidence in its safety and efficacy before offering it to millions of people. For example, the FDA frequently requires a finding of efficacy in two phase 3 trials before approving medical interventions for marketing to the public.

Still, a finding of safety and efficacy in a phase 3 trial is an ethically critical point in the vaccine development process. From that point, receiving the vaccine candidate is known to offer protection to many individuals. Hence, receiving a placebo instead is contrary to their interests, even though it may be necessary to collecting socially valuable data.

This tension between protecting research participants and gathering data that may benefit others is common to clinical research, and there is substantial literature on how to address it (7). Although this literature applies to the ethics of vaccine trials, the present situation is unparalleled.

There is a global crisis for which there are currently limited treatment options. Hence, billions of individuals could benefit from a safe and efficacious vaccine. And having so many vaccine candidates in development substantially increases the chances that one or more will be found to be safe and efficacious while others are still being tested. Limitations on current treatment options mean that it is in each individual's interests to receive the first vaccine found to be safe and efficacious, rather than participate in vaccine trials where they might receive placebo or an unproven vaccine candidate.

In addition, given the challenges of manufacturing sufficient supply for so many people, and given that different vaccine candidates may be efficacious in different populations, several vaccines may be needed to meet the global need. This highlights the potential social value of conducting additional trials after one or more vaccine candidates are found to be safe and efficacious.

Moreover, vaccine candidates typically have not been widely available until after they receive marketing approval by the FDA. However, the FDA has indicated that it may take the unprecedented step of making a vaccine candidate that is found to be safe and efficacious widely available through an emergency use authorization (EUA) before marketing approval. An EUA involves the FDA commissioner allowing 
unapproved medical products to be used in an emergency to diagnose, treat, or prevent serious or life-threatening diseases or conditions "when there are no adequate, approved, and available alternatives" (8).

\section{PARTICIPANTS, NOT PATIENTS}

Pfizer recently announced that at the first interim analysis, its vaccine candidate was found to be $95 \%$ effective at preventing symptomatic COVID-19 $(4,5)$. A week later, Moderna announced that its vaccine candidate was found to be $94.5 \%$ effective. At that point, the trials had enrolled more than 43,000 and 30,000 participants, respectively, of whom 94 and 95 developed symptomatic disease. If these findings are confirmed, they will represent an important public health advance, and provision of the vaccine candidates would be in the interests of many individuals. The FDA may thus grant an EUA, making either or both vaccines available before each study's completion and also before FDA marketing approval.

Some commentators argue that clinical trials are ethically acceptable only as long as there is insufficient evidence that the intervention offered in one arm is superior to what is offered in another arm, or to what is available outside the trial (9). This view implies that it would be unethical to continue Pfizer's and Moderna's placebo-controlled trials given the findings of efficacy. It also implies it would be unethical to test other unproven vaccine candidates against placebo.

Proponents defend this view on the grounds that it provides a way to protect participants from excessive risks. They argue further that researchers conducting clinical trials are obligated to treat participants consistent with their clinical interests and conclude that it is unethical to give participants a placebo once a safe and efficacious vaccine has been identified.

We disagree. This view fails to recognize that the obligations researchers have to their participants are distinct from the obligations that clinicians have to their patients (9). It further ignores the existence of other mechanisms, including independent review and informed consent, to protect research participants from excessive risks. The differences between the ethics of clinical research and clinical care are reflected in the consensus that it can be ethically appropriate to invite research participants to accept some risks to collect socially valuable data. In particular, codes and guidelines around the world permit researchers to expose participants in clinical trials, including vaccine trials, to some risks to collect socially valuable data that cannot be obtained in a less risky way (10).

These guidelines have a critical implication for COVID-19 vaccine trial ethics: Researchers are not obligated to provide placebo recipients with a safe and efficacious vaccine once one has been identified. Instead, researchers are obligated to ensure that any plans to conduct placebo-controlled trials remain ethically appropriate given current evidence. Such plans must be reviewed and approved by an independent board, known as an institutional review board (IRB) in the United States, which must find that a number of conditions are satisfied (11). Two of these conditions are particularly important for determining the acceptability of conducting placebo-controlled trials after a vaccine candidate has been found to be safe and efficacious: Is the trial's risk-benefit profile still acceptable? Do participants consent?

\section{ACCEPTABLE RISK-BENEFIT PROFILE}

Continuing a blinded, placebo-controlled trial after the vaccine candidate being tested has been found to be safe and efficacious is justified only when the trial's risk-benefit profile remains acceptable. Making this determination requires answering three questions: (i) What is the social value of continuing the trial? (ii) What are the risks to participants of continuing the trial? (iii) Are the risks to participants sufficiently low and justified by the social value of continuing the trial?

\section{Social value}

Continuing a trial after the vaccine candidate has been found to be safe and efficacious can provide an opportunity to collect several types of socially valuable data. Most importantly, it can provide greater confidence in and a more precise point estimate of the vaccine's efficacy and offer an opportunity to collect additional safety data, including data on any uncommon or delayed side effects. It can also help to assess how long the vaccine's protective effect lasts; offer insight into the vaccine's impact in various subgroups, such as older individuals or those with comorbidities; and evaluate whether the vaccine candidate protects against infection itself.

\section{Risks to participants}

Once a vaccine candidate is found to be efficacious, participants in the placebo arm are known to be at higher risk of symptomatic disease than those in the active arm. The degree of risk depends on the chances that participants in the placebo arm will become infected, the risks they face if they are, and how much protection the vaccine candidate offers.

The chances that participants in the placebo arm will be infected depends on the local transmission rate, preventive measures they adopt, and the amount of time they remain on placebo. When participants are on placebo for a short time, the chances of infection are correspondingly low. For example, Moderna plans to continue its placebo-controlled trial until 151 cases of symptomatic disease are identified (5). Given current transmission rates, this may take as little as a few weeks.

Remaining on placebo for a few weeks, rather than accessing an efficacious vaccine, poses a low chance of substantial harm. Continuing on placebo for even longer periods also 
poses a low chance of substantial harm to individuals at low risk for severe disease.

Remaining on placebo for an extended period can pose considerable risks to individuals at high risk of severe disease. The extent of these risks depends critically on what options are available to them. In the setting of few effective treatments and potentially strained hospital systems, receiving placebo for an extended period rather than a safe and efficacious vaccine can pose substantial risks. However, if highrisk individuals would not have access to a safe and efficacious vaccine outside of research-for example, there is only enough supply for the trial or they are not part of a prioritized group that will receive the vaccine during the time of the trial-receiving placebo in a clinical trial poses few additional risks to them.

\section{Are risks sufficiently low and justified?}

There is no algorithm for determining how much social value a given clinical trial has and whether its social value justifies the risks participants face. As a result, IRBs tend to focus on ensuring that a trial has the potential to collect important data and that the risks of substantial harm are low.

Trials with the potential to collect data helpful for addressing a global pandemic have considerable social value. Inviting competent adults to participate in such trials can be ethical when doing so poses a small increase in their risk of experiencing substantial harm. This suggests that it can be ethically acceptable to continue a placebo-controlled trial for a short period after the vaccine candidate has been found to be safe and efficacious, even when participants might be able to access the vaccine candidate outside the trial, for example, through an EUA (12). By contrast, if continuing the trial does not offer the opportunity to collect socially valuable data, or comparable data can be obtained in less risky ways, continuing the trial with a placebo arm for any length of time would be ethically problematic.

Inviting participants who are at low risk of severe disease to remain blinded and stay in the trial for a longer period can be acceptable when it offers the potential to collect data that might be helpful for addressing the pandemic. In most cases, continuing a blinded, placebo-controlled design with highrisk individuals for longer periods will not yield data of sufficient value to justify it. Exceptions might include when the individuals cannot access an efficacious vaccine outside the trial and their participation is needed to collect valuable data, or they are in a group for whom no efficacious vaccine candidate has been identified. Otherwise, individuals at high risk of severe disease should be unblinded and those on the placebo arm offered the vaccine within a redesigned study or given the opportunity to seek the vaccine outside the trial.

When the value of the data to be collected does not justify the risks of continuing the trial as designed, researchers have several options. They can unblind participants; offer placebo recipients the vaccine, possibly as part of an expanded access program; and follow them to collect additional data (13). Alternatively, researchers might redesign the trial, for example, to include a crossover in which the blind is maintained and those on the placebo arm receive the vaccine after they complete the placebo arm. Finally, in some cases, it may make sense to simply stop the trial and unblind participants, thus allowing those in the placebo arm to seek the vaccine elsewhere.

\section{OTHER TRIALS}

Even when a vaccine candidate is found to be safe and efficacious, there are likely to be good reasons to study others. Another vaccine candidate might be more effective, generate longer-lasting immunity, work better in certain subpopulations, provide greater protection against severe disease, or prevent infection better. Other candidates may also be superior with respect to cost or other practical considerations. For example, Pfizer's vaccine candidate must be stored at very low temperatures until 5 days before administration, a requirement which will reduce its availability in many parts of the world.

When there are safe and effective interventions available, the default is to use them rather than placebo as the comparator in clinical trials. Thus, if the Pfizer or Moderna vaccine candidates, or others, are confirmed to be safe and efficacious, researchers should consider whether their trials can be redesigned as a comparison between the vaccine candidate they are testing and a safe and efficacious vaccine. When making this decision, researchers and IRBs should take into account that an active comparison trial is likely to require larger sample sizes and extend the duration of the trial.

Redesigning ongoing trials to include an efficacious vaccine as an active comparator may not always be feasible or valuable. If continuing to evaluate the vaccine candidate nonetheless offers important social value, participants at low risk of severe disease might be invited to stay blinded and remain in the placebo-controlled trial. If continued participation appreciably increases participants' risk of severe disease, researchers should discuss participants' options for obtaining an efficacious vaccine outside research.

\section{DISCLOSURE, UNBLINDING, CONSENT}

Researchers are ethically obligated to inform participants of developments that might influence their willingness to remain in a clinical trial. Clearly, the fact that a vaccine candidate has been found to be safe and efficacious meets this standard. Hence, investigators should inform participants in all trials of such a finding. This information should include the vaccine's safety record, the level of protection it provides, the populations for which it has been found to be safe and 
efficacious, and whether it might be available through an EUA or other means.

Researchers should then explain the plans for their trial going forward and solicit participants' consent. To minimize potential confusion and distrust (14), participants should understand the rationale behind the plan and that their prior consent does not obligate them to continue to participate. If researchers intend to continue with a placebo-controlled design, participants can be encouraged to remain in the trial. However, once a safe and efficacious vaccine candidate is available, knowing whether they received placebo or vaccine becomes relevant to a participant's decision whether to seek the vaccine outside research. Hence, participants who choose to leave the trial should be informed whether they received the vaccine candidate or placebo.

If one or more efficacious vaccines become widely available outside research, continuing a blinded, placebo-controlled trial might result in participants in both the active and placebo arms dropping out and seeking the vaccine elsewhere. Researchers should anticipate this possibility and consider whether they might unblind participants, offer an efficacious vaccine to the placebo recipients, and follow everyone. Alternatively, it might make sense to continue the blinded, placebo-controlled trial with a guarantee that individuals in the placebo arm will receive an efficacious vaccine once their participation in the study is completed. With careful planning and systematic assessment of the social value and risks, we believe it can be ethical to conduct some placebo-controlled trials of vaccine candidates for COVID-19 even after we have an efficacious vaccine. Doing so may be necessary to effectively address a pandemic that is causing so much harm around the world.

\section{REFERENCES AND NOTES}

1. Bioworld, Biopharma products in development for COVID-19 (2020); www.bioworld.com/COVID19products.

2. J. Corum, S. L. Wee, C. Zimmer, Coronavirus vaccine tracker (2020); www.nytimes.com/interactive/2020/science/coronavirus-vaccinetracker.html.

3. FDA, "Development and licensure of vaccines to prevent COVID-19: Guidance for industry" (FDA-2020-D-1137, FDA, 2020); www.fda.gov/media/139638/download.

4. Pfizer, Pfizer and BioNTech announce vaccine candidate against COVID-19 achieved success in first interim analysis from phase 3 study, 9 November 2020; www.pfizer.com/news/press-release/press-release-detail/pfizer-and-biontechannounce-vaccine-candidate-against.

5. Moderna, Moderna's COVID-19 vaccine candidate meets its primary efficacy endpoint in the first interim analysis of the phase 3 COVE study, 16 November 2020; https://investors.modernatx.com/news-releases/news-releasedetails/modernas-covid-19-vaccine-candidate-meets-its-primary-efficacy.

6. P. Doshi, BMJ 371, m4037 (2020). doi:10.1136/bmi.m4037 Medline

7. J. Millum, C. Grady, Contemp. Clin. Trials 36, 510 (2013). doi:10.1016/j.cct.2013.09.003 Medline

8. FDA, Emergency use authorization (2020); www.fda.gov/emergencypreparedness-and-response/mcm-legal-regulatory-and-policyframework/emergency-use-authorization.

9. S. P. Hey, A. J. London, C. Weijer, A. Rid, F. Miller, BMJ 359, j5787 (2017). doi:10.1136/bm.j.j5787 Medline
10. Council for International Organizations of Medical Sciences (CIOMS), "International ethical guidelines for health-related research involving humans" (Report, CIOMS, 2016); https://cioms.ch/wp-content/uploads/2017/01/WEBCIOMS-EthicalGuidelines.pdf.

11. E. J. Emanuel, D. Wendler, C. Grady, JAMA 283, 2701 (2000). doi:10.1001/iama.283.20.2701 Medline

12. FDA, Emergency use authorization for vaccines to prevent COVID-19 guidance for industry (2020); www.fda.gov/media/142749/download.

13. M. W. Mccarthy, D. Oshinsky, A. Caplan, "Make pre-approval Covid-19 vaccines available through expanded access, not an EUA," Stat, 11 November 2020; www.statnews.com/2020/11/09/expanded-access-not-eua-for-distributingpreapproval-covid-19-vaccines/.

14. R. C. Warren, L. Forrow, D. A. Hodge Sr., R. D. Truog, N. Engl. J. Med. 10.1056/NEJMp2030033 (2020).

\section{ACKNOWLEDGMENTS}

Thanks to E. Erbelding for valuable discussion of this issue. This work was funded by the Intramural Research Program at the U.S. National Institutes of Health Clinical Center. The authors declare no competing interests. Disclaimer: The opinions expressed are the authors' own. They do not represent the position or policy of the National Institutes of Health, the U.S. Public Health Service, or the U.S. Department of Health and Human Services.

Published online 3 December 2020

10.1126/science.abf5084 